\title{
Case Report: Fatal myocarditis after combination of immune checkpoint inhibitor and platinum doublet chemotherapy
}

\section{[version 1; peer review: 1 not approved]}

\author{
Vilde Drageset Haakensen (iD1, Solfrid M.H. Thunold1', Geeta Gulati2 \\ ${ }^{1}$ Department of Oncology, Oslo University Hospital, P.O. Box 4956 Nydalen, Oslo, 0424, Norway \\ ${ }^{2}$ Department of Cardiology, Oslo University Hospital, P.O. Box 4956 Nydalen, Oslo, 0424, Norway
}

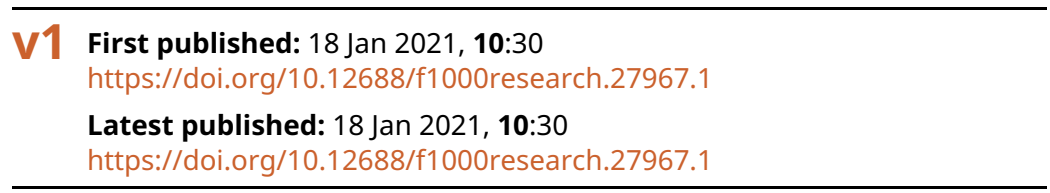

\section{Abstract}

Myocarditis is a rare but feared adverse event of treatment with immune checkpoint inhibitors. The incidence is about $1 \%$, while the mortality rate is up to $50 \%$. Many physicians treating lung cancer do not have experience with this serious adverse event, and few hospitals in Scandinavia have routines for baseline assessments that may help detect early signs of inflammation of the myocard. Early onset of antiinflammatory treatment is associated with favourable outcome. We present a case report of fatal myocarditis after treatment with check-point inhibitor. The patient was admitted with severe dyspnoea, general body ache, weakness, dysphagia, palpitations and diplopia two weeks after her second infusion of systemic treatment combining chemotherapy and immunotherapy. She had presented vague symptoms at time of the second infusion that were not identified as related to myocarditis/myositis. Upon aggressive treatment with methylprednisolone, mycophenolate mofetil, abatacept and plasmapheresis, her troponins and pro-BNP were reduced, but clinically she deteriorated and her life could not be saved. We present this case report to increase awareness of the condition and to raise discussion about the role of routine baseline assessments to aid early diagnosis and anti-inflammatory treatment to prevent treatment-related deaths.

\section{Keywords}

myocarditis, myositis, immunotherapy, checkpoint inhibitor, adverse events

\section{Open Peer Review \\ Approval Status $X$ \\ 1 \\ version 1 \\ 18Jan 2021 \\ $\underset{\text { view }}{\mathrm{X}}$ \\ 1. Panagiotis T. Diamantopoulos (D), National and Kapodistrian University of Athens, \\ Athens, Greece \\ Any reports and responses or comments on the article can be found at the end of the article.}


Corresponding author: Vilde Drageset Haakensen (vilde.haakensen@gmail.com)

Author roles: Haakensen VD: Conceptualization, Data Curation, Writing - Original Draft Preparation; Thunold SMH: Data Curation; Gulati G: Conceptualization, Data Curation, Methodology, Writing - Original Draft Preparation

Competing interests: Vilde Drageset Haakensen has received honorary lectures and advisory boards from Takeda, Astra Zeneca, Pfizer, BMS, Roche. Geeta Gulati has received honorary lectures for BMS and Astra Zeneca.

Grant information: The author(s) declared that no grants were involved in supporting this work.

Copyright: $\odot 2021$ Haakensen VD et al. This is an open access article distributed under the terms of the Creative Commons Attribution License, which permits unrestricted use, distribution, and reproduction in any medium, provided the original work is properly cited.

How to cite this article: Haakensen VD, Thunold SMH and Gulati G. Case Report: Fatal myocarditis after combination of immune checkpoint inhibitor and platinum doublet chemotherapy [version 1; peer review: 1 not approved] F1000Research 2021, 10:30 https://doi.org/10.12688/f1000research.27967.1

First published: 18 Jan 2021, 10:30 https://doi.org/10.12688/f1000research.27967.1 


\section{Introduction}

Myocarditis is a rare but feared adverse event of treatment with immune checkpoint inhibitors (ICI). The incidence is about $1 \%$, while the mortality rate is up to $50 \%$. The incidence of lung cancer in Norway is 117 per 100000 with 3200 patients a year. $30 \%$ of patients are treated with surgery or stereotactic radiation. The remaining patients are eligible to treatment with ICI in first or later lines. ICI has been used for lung cancer in Norway since 2016. Still, most physicians in Norway treating lung cancer have not experienced ICI-induced myocarditis. An informal query to six university hospitals and eight local hospitals in the South-East Health Region in Norway revealed no routine examination (blood or imaging) at baseline to help detect development of ICI-induced myocarditis later. An electrocardiogram (ECG) is usually part of the diagnostic workup before cancer treatment, but only two hospitals specify taking ECG before start of immunotherapy in later lines. Our impression is that the situation is similar in the other Nordic countries (after personal communication through the Nordic Cardio-Oncology Board). The literature indicates the importance of early diagnosis, as a retrospective study found favourable outcome with onset of corticosteroid treatment within $24 \mathrm{hrs}$ of admission to hospital ${ }^{1}$. We present this case to increase awareness, to advocate early diagnosis and treatment of ICI-related myocarditis.

\section{Case}

A woman in her late sixties, working freelance without any exposure and never smoking, with paroxysmal atrial fibrillation and a subvalvular aortic stenosis had a right upper lung lobectomy for an epidermal growth factor receptor (EGFR) positive adenocarcinoma. Post-surgery, she was treated with adjuvant chemotherapy and radiotherapy. A relapse two years later was treated with erlotinib and paused after a year due to gradual progression. After 18 months, upon progression, carboplatin/ pemetrexed/pembrolizumab was started. When seen for the second infusion, she had slightly increased dyspnoea and was feeling tender on palpation of the chest wall medial of her right breast and the food felt as if it stopped in her oesophagus if she ate too quickly. There was no cough or fever. Clinical examination, c-reactive protein (CRP), haematology and electrolytes were normal. Troponins and creatine kinase $(\mathrm{CK})$ were not measured. Computerized tomography (CT) showed no signs of pneumonitis and carboplatin/pemetrexed/pembrolizumab treatment was continued.

Two weeks later she was admitted to the oncology department with increased dyspnoea, general body ache, muscular weakness, dysphagia, episodes of palpitations and diplopia. Clinical examination revealed ptosis. Her blood work-up showed troponin T $651(<14)$ ng/L, NT-proBNP $6761(<760)$ ng/L, CRP $6.4(<5) \mathrm{mg} / \mathrm{L}$, aspartate aminotransferase (AST) $453(<35) \mathrm{U} / \mathrm{L}$ (grade 3 toxicity), alanine aminotransferase (ALT) $186(<45) \mathrm{U} / \mathrm{L}$ (grade 2), lactate dehydrogenase (LDH) 1111 (<255) U/L and creatine kinase (CK) $5845 \quad(<210)$ U/L. Her ECG showed sinus rhythm and a new left bundle branch block (Figure 1A). CT excluded pulmonary embolism. Three days later she developed a third-degree atrioventricular block (Figure 1B) and her clinical symptoms progressed. Echocardiography showed no regional wall motion abnormalities. Cardiac magnetic resonance imaging (CMR) was attempted, but aborted as the patient was unstable because of her third-degree atrioventricular block. A two-chamber pacemaker was implanted and a coronary angiography was done and showed no signs of acute coronary syndrome. Later, repeated echocardiography was unchanged from the initial echocardiography, the left ventricular ejection fraction (LVEF) continued to be normal of $58 \%$. With the presenting symptoms, troponin elevation, third degree atrioventricular block and no significant stenosis on the coronary angiogram, the patient fulfilled the criteria of myocarditis ${ }^{2}$ and it was concluded the patient had ICI-induced myocarditis with myositis and myasthenia gravis-like syndrome.

Intravenous methylprednisolone $2 \mathrm{mg} / \mathrm{kg} /$ day was initiated on day 3 and increased to $1000 \mathrm{mg} /$ day for four days as her troponins increased (Figure 2). As there was a lack of clinical and biochemical response, abatacept $1000 \mathrm{mg}$ once every second week and oral mycophenolate mofetil $750 \mathrm{mg}$ b.i.d was initiated. This improved the biochemical parameters, but the clinical symptoms were unchanged. On day 14 plasmapheresis with exchange of 21 plasma was performed, but could not be repeated as the patient did not tolerate the exchange. However, the biochemical values improved further and her ptosis, dyspnoea and general muscle weakness improved. Unfortunately, her dysphagia and dyspnoea kept deteriorating. On day 25, abatacept and mycophenolate mofetil was stopped and the patient died five days later.

\section{Discussion}

Myocarditis is a rare incidence $(0.06-1.14 \%)^{3,4}$ off-target effect of ICI with mortality rates up to $50 \%{ }^{5}$. The symptoms are often non-specific as fatigue, dyspnoea or chest pain. In severe cases cardiac arrhythmias as atrioventricular block or ventricular arrhythmias can occur. The myocarditis can be combined with myositis and/or myasthenia gravis like syndrome ${ }^{6}$. With increasing use of immunotherapy alone and in combination with other therapies, an increase in immune mediated myocarditis can be expected. Combination immunotherapy has a higher incidence than distribution of a PD-1/PD-L1 inhibitor alone $^{7}$. There are few studies reporting the incidence of myocarditis after combination of ICI and chemotherapy. Keynote 189 reported one case of myocarditis in the treatment arm with pembrolizumab, carboplatin and pemetrexed (of 405 patients) ${ }^{8}$.

As the incidence of myocarditis is rare, the symptoms non-specific and because in the acute setting, the patients are often seen by medical staff with less experience of immunotherapy off-target effects, the diagnosis may be missed unless there are routines for baseline examinations and pre-defined early diagnostic work-ups. As a minimum, before initiation of immunotherapy, ECG should be taken and troponins and NT-proBNP measured. There should be a low threshold for repeating these measures during treatment, particularly if the patient is admitted to the hospital. After onset of symptoms suspicious of myocarditis, coronary angiography can rule out acute coronary syndrome, while CMR can often detect 
A

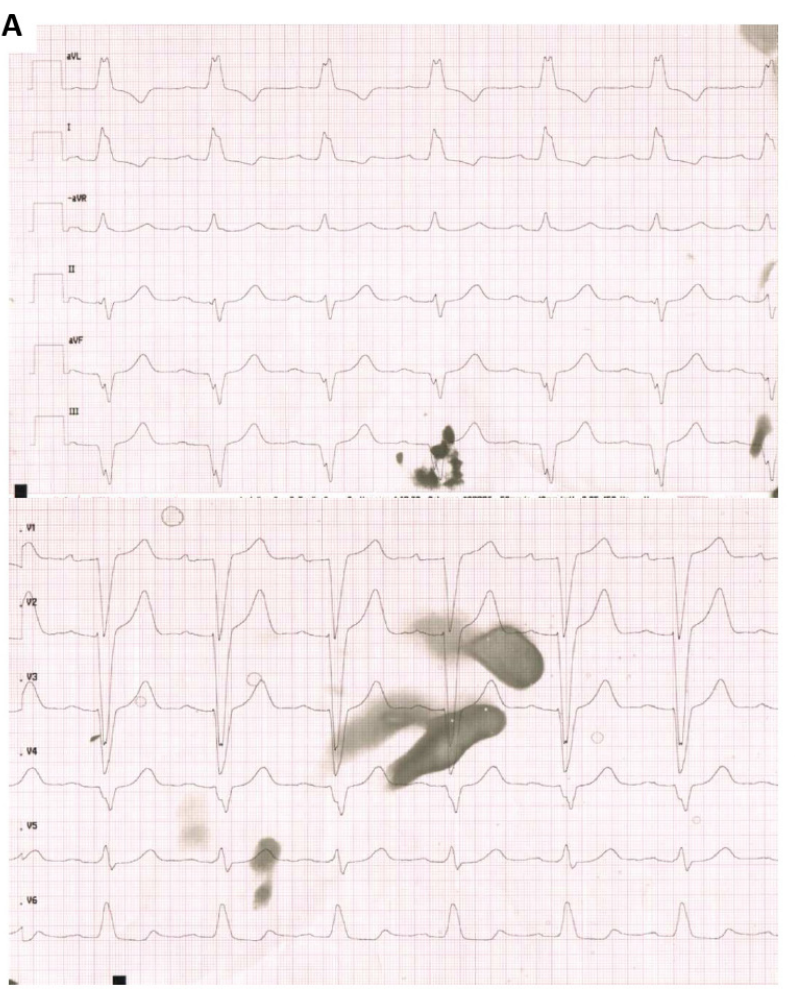

\section{B}

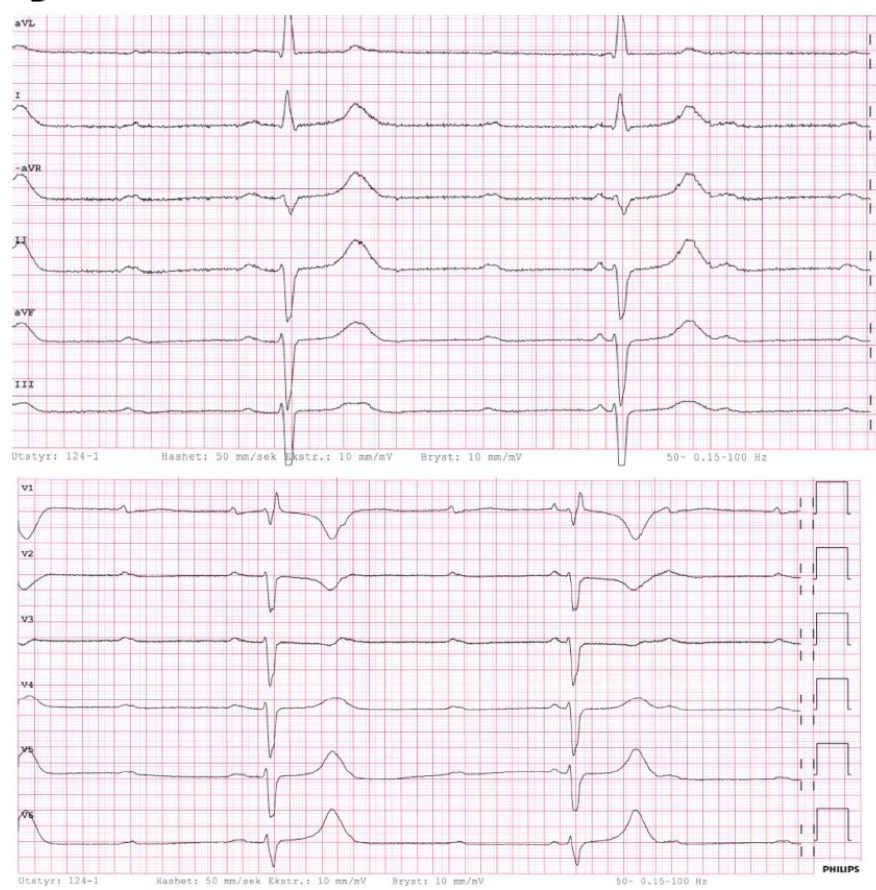

Figure 1. Electrocardiogram at admission after the second infusion of combination therapy (A) and three days after admission (B).

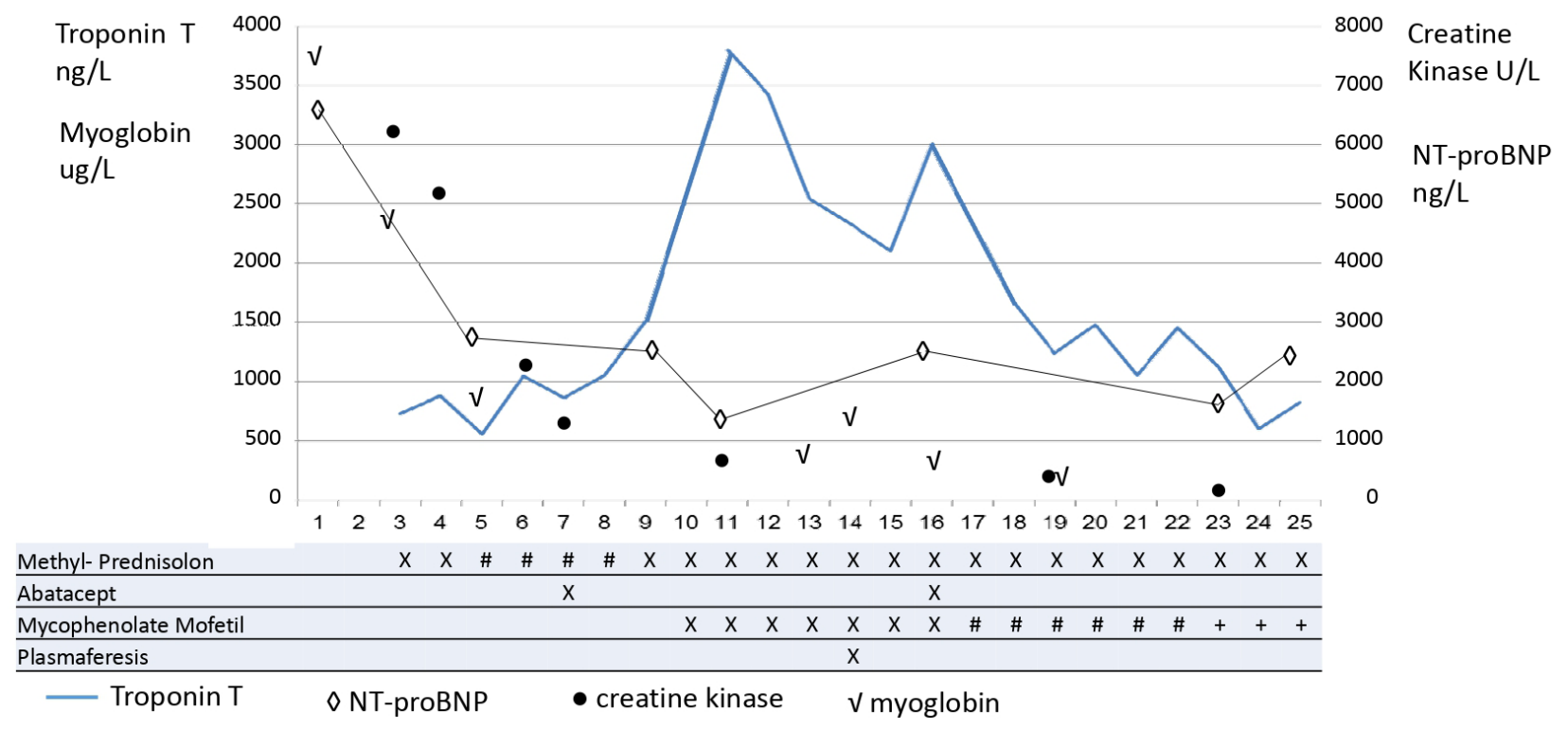

Figure 2. Serum markers of myocardial and muscular damage during hospitalization. Serum levels of troponin T increase during the first 10 days. Normalization of creatine kinase and myoglobin may reflect improvement of myositis, while the myocarditis did not resolve. Daily treatment regimens are indicated as follows: methylprednisolone X= 2 mg/kg, \#= $1000 \mathrm{mg} \times 1$; abatacept X=20 mg/kg; mycophenolate mofetil X= $750 \mathrm{mg} \times 2, \#=500 \mathrm{mg} \times 2,+=500 \mathrm{mg} \times 1$. 
myocardial oedema along with late gadolinium contrast enhancement (LGE) as a sign of inflamed myocardium. However, in a recent study of 103 patients with ICI-associated myocarditis LGE was only present in $48 \%$, other signs of oedema as elevated T2-weighted short tau inversion recovery (STIR) were present in $28 \%$. The presence of LGE or T2-weighted STIR were not associated with major adverse cardiovascular events (composite of cardiovascular death, cardiogenic shock, cardiac arrest and complete heart block) ${ }^{9}$. In our patient CMR was not performed as she was unstable because of a third-degree atrioventricular block and implantation of a pacemaker was prioritized. Diagnosis was based on the diagnostic criteria for clinically suspected myocarditis as recommended by the European Society of Cardiology $(\mathrm{ESC})^{2}$. Early immune suppression is of the essence, and hence, clinical awareness and diagnostic routines are important. In a recent retrospective study, high dose methylprednisolone $(1000 \mathrm{mg} / \mathrm{d})$ and early initiation $(<24 \mathrm{~h})$ were associated with improved cardiac outcomes $^{1}$. Combination of several anti-inflammatory therapies and plasmapheresis may reverse the serious adverse event ${ }^{6,10}$.

\section{Conclusion}

Education of medical staff in various hospital departments and in the community is necessary and may increase awareness of ICI-associated myocarditis. Clear routines for standard investigations prior to and during treatment with immunotherapy may reduce time to diagnosis, increase awareness and hopefully reduce mortality of myocarditis.

\section{Data availability}

All data underlying the results are available as part of the article and no additional source data are required.

\section{Consent}

Written informed consent for publication of their clinical details and/or clinical images was obtained from the patient.
1. Zhang L, Zlotoff DA, Awadalla M, et al.: Major adverse cardiovascular events and the timing and dose of corticosteroids in immune checkpoint inhibitor-associated myocarditis. Circulation. 2020; 141(24): 2031-4. PubMed Abstract | Publisher Full Text | Free Full Text

2. Caforio ALP, Pankuweit S, Arbustini E, et al.: Current state of knowledge on aetiology, diagnosis, management, and therapy of myocarditis: A position statement of the European Society of Cardiology Working Group on Myocardial and Pericardial Diseases. Eur Heart J. 2013; 34(33): 2636-48, 2648a-2648d.

PubMed Abstract | Publisher Full Text

3. Martins F, Sofiya L, Sykiotis GP, et al.: Adverse effects of immune-checkpoint inhibitors: epidemiology, management and surveillance. Nat Rev Clin Oncol. 2019; 16(9): 563-80.

PubMed Abstract | Publisher Full Text

4. Haanen JBAG, Carbonnel F, Robert C, et al:: Management of toxicities from immunotherapy: ESMO Clinical Practice Guidelines for diagnosis, treatment and follow-up. Ann Oncol. 2017; 28(suppl_4): iv119-iv142. PubMed Abstract | Publisher Full Text

5. Salem JE, Manouchehri A, Moey M, et al.: Cardiovascular toxicities associated with immune checkpoint inhibitors: an observational, retrospective, pharmacovigilance study. Lancet Oncol. 2018; 19(12): 1579-1589. PubMed Abstract | Publisher Full Text | Free Full Text

6. Hu J, Florido R, Lipson EJ, et al.: Cardiovascular toxicities associated with immune checkpoint inhibitors. Cardiovasc Res. 2019; 115(5): 854-68. PubMed Abstract | Publisher Full Text | Free Full Text

7. Mahmood SS, Fradley MG, Cohen J V, et al.: Myocarditis in Patients Treated With Immune Checkpoint Inhibitors. J Am Coll Cardiol. 2018; 71(16): 1755-64. PubMed Abstract | Publisher Full Text | Free Full Text

8. Gadgeel S, Rodríguez-Abreu D, Speranza G, et al.: Updated Analysis From KEYNOTE-189: Pembrolizumab or Placebo Plus Pemetrexed and Platinum for Previously Untreated Metastatic Nonsquamous Non-Small-Cell Lung Cancer. J Clin Oncol. 2020; 38(14): 1505-17. PubMed Abstract | Publisher Full Text

9. Zhang L, Awadalla M, Mahmood SS, et al:: Cardiovascular magnetic resonance in immune checkpoint inhibitor-associated myocarditis. Eur Heart J. 2020; 41(18): 1733-43.

PubMed Abstract | Publisher Full Text | Free Full Text

10. Salem JE, Allenbach $Y$, Kerneis M: Abatacept for severe immune checkpoint inhibitor-associated myocarditis. N EnglJ Med. 2019; 380(24): 2377-2379. PubMed Abstract | Publisher Full Text 


\section{Open Peer Review}

\section{Current Peer Review Status: $\mathrm{X}$}

\section{Version 1}

Reviewer Report 15 February 2021

https://doi.org/10.5256/f1000research.30933.r79530

(c) 2021 Diamantopoulos P. This is an open access peer review report distributed under the terms of the Creative Commons Attribution License, which permits unrestricted use, distribution, and reproduction in any medium, provided the original work is properly cited.

\section{Panagiotis T. Diamantopoulos}

First Department of Internal Medicine, Laikon General Hospital, National and Kapodistrian University of Athens, Athens, Greece

It was a pleasure to review this interesting case report by Haakensen et al. on the emergence of a complex neurologic/cardiac irAE in a patient with lung adenocarcinoma treated with pembrolizumab. Although the case is interesting, there are already several case reports in the literature on the emergence of such irAEs in patients treated with checkpoint inhibitors. Moreover, the investigation of the patient is somewhat incomplete, thus a definitive diagnosis cannot be made with accuracy. You will find my comments in detail below.

\section{Major points}

Introduction, paragraph 1 lines 8-20. The information provided here is mainly hypothetical and the methodology of the authors does not follow any scientific research rules. I suggest it to be removed. In any case it does not add to the manuscript.

English editing by a native speaker is highly recommended.

Case, paragraph 1, lines 9-13. The description of the symptoms and signs of the patient should be more accurate. Moreover, medical terms such as dysphagia/odynophagia etc. should be used. Moreover, the findings of a detailed neurologic examination should be provided. Since there are clear signs of neurologic deterioration, did the treating doctors investigate the patient with a brain CT/MRI or with a lumbar puncture?

Case. I suggest that the authors provide figures of CT scans, echocardiography, and coronary angiography.

Why did the doctors stop immunosuppression 5 days before the death of the patient? More information is needed on the decision.

Did the treating doctors administer pyridostigmine to the patient, especially since the 
authors correctly identified the condition as myasthenia gravis with cardiac involvement? How did they support the diagnosis? Did they measure anti-AChR antibodies? I believe that the diagnosis of myasthenia gravis, although obvious, should be further supported.

Does the very low incidence of cardiac irAEs justify the measurement of troponin and NTproBNP before treatment initiation, as suggested by the authors (Discussion, paragraph 2, lines 7-8)? The authors should provide relevant references if available.

Data availability: the authors should state that upon reasonable request the presented data is available.

Since this is a case of concomitant development of neurologic and cardiac irAEs, the authors should reference some relevant studies such as those by Suzuki S et al. "Nivolumab-related myasthenia gravis with myositis and myocarditis in Japan" ${ }^{1}$ or my own paper, Diamantopoulos PT et al. "Concomitant development of neurologic and cardiac immune-related adverse effects in patients treated with immune checkpoint inhibitors for melanoma"2.

\section{Minor points}

Introduction, paragraph 1, line 4. "3200 new cases per year" instead of "3200 patients a year".

Introduction, paragraph 1, line 5. The sentence should not start with a number - please rephrase.

Case, paragraph 1, line 1. What does "working freelance without any exposure" mean?

Case, paragraph 1, line 7. "And paused" seems like referring to the relapse. Please rephrase.

Case, paragraph 1, line 8. What does "gradual progression" mean? Please provide more information and CT scan pictures at baseline and at disease progression.

\section{References}

1. Suzuki S, Ishikawa N, Konoeda F, Seki N, et al.: Nivolumab-related myasthenia gravis with myositis and myocarditis in Japan.Neurology. 2017; 89 (11): 1127-1134 PubMed Abstract | Publisher Full Text

2. Diamantopoulos PT, Tsatsou K, Benopoulou O, Bonou M, et al.: Concomitant development of neurologic and cardiac immune-related adverse effects in patients treated with immune checkpoint inhibitors for melanoma.Melanoma Res. 30 (5): 484-491 PubMed Abstract | Publisher Full Text

Is the background of the case's history and progression described in sufficient detail? No

Are enough details provided of any physical examination and diagnostic tests, treatment given and outcomes?

No

Is sufficient discussion included of the importance of the findings and their relevance to future understanding of disease processes, diagnosis or treatment? 
No

Is the case presented with sufficient detail to be useful for other practitioners?

No

Competing Interests: No competing interests were disclosed.

Reviewer Expertise: Melanoma, Immunotherapy, myelodysplastic syndrome

I confirm that I have read this submission and believe that I have an appropriate level of expertise to state that I do not consider it to be of an acceptable scientific standard, for reasons outlined above.

Author Response 08 Mar 2021

Vilde Drageset Haakensen, Oslo University Hospital, P.O. Box 4956 Nydalen, Oslo, Norway

We thank Dr Diamantopoulos for reviewing our case report. The first aim of this report was to increase clinicians' awareness of immune checkpoint inhibitor (ICI) induced myocarditis/myositis. Even though case reports of ICI myocarditis are found and recommendations on how to follow-up and treat these patients are published, clinically the routine of cardiac baseline examination and follow-up could be improved. The introduction is based on statistics. The experiences gathered from Scandinavian colleagues are used to emphasize the lack of cardiovascular baseline stratification of these patients in the clinical setting.

The second aim was to discuss adequate measures to prevent fatal outcomes by discussing the use of baseline and follow-up troponin and proBNP. The use of these biomarkers and other surveillance tools have already been justified in a Position statement from the CardioOncology Study Group of the Heart Failure Association and the Cardio-Oncology Council of the European Society of Cardiology (Pudil et al. Eur J Heart Fail. 2020).

The patient was not examined by brain CT/MRI or lumbar puncture or anti-AChR measurements and pyridostigmine was not used. We agree that neurologists should be involved in such cases and that reference from the neurology field would improve the discussion.

Steroids were stopped five days before the patient died as she was clinically deteriorating and not tolerating the aggressive immunosuppressive therapy. We agree that additional imaging of the heart and tumor would enhance the quality of this case.

Competing Interests: No competing interests were disclosed.

Author Response 25 Mar 2021

Vilde Drageset Haakensen, Oslo University Hospital, P.O. Box 4956 Nydalen, Oslo, Norway We do not wish for additional reviews of this case report. 
Competing Interests: No competing interests were disclosed.

The benefits of publishing with F1000Research:

- Your article is published within days, with no editorial bias

- You can publish traditional articles, null/negative results, case reports, data notes and more

- The peer review process is transparent and collaborative

- Your article is indexed in PubMed after passing peer review

- Dedicated customer support at every stage

For pre-submission enquiries, contact research@f1000.com 